\title{
UNDECIDABILITY OF THE THEORY OF ABELIAN GROUPS WITH A SUBGROUP
}

\author{
WALTER BAUR 1
}

ABSTRACT. The theory of abelian groups with an additional predicate denoting a subgroup is undecidable.

0 . Introduction. Let $L$ be the first order language with nonlogical symbols 0 , ,$+ P$, where $P$ is a unary predicate symbol. For any class $K$ of abelian groups let $T(K)$ denote the $L$-theory of the class of structures $\langle A, B\rangle$, where $A \in K$ and $B \subseteq A$ is an arbitrary subgroup. Kozlov and Kokorin [4] showed that $T(K)$ is decidable if $K$ is the class of torsion free groups. The main result of this paper is the following:

THEOREM 1. Let $p$ be a prime number and let $K$ be the class of abelian groups $A$ such that $p^{9} A=0$. Then $T(K)$ is undecidable.

An immediate consequence is

COROllary 2. T(class of all abelian groups) is undecidable.

Corollary 2 answers a few questions asked in [4].

1. Proof of Theorem 1. Let $S$ be a finitely presented semigroup on two generators $\alpha_{1}, \alpha_{2}$ and defining relations $V_{\nu}\left(\alpha_{1}, \alpha_{2}\right)=W_{\nu}\left(\alpha_{1}, \alpha_{2}\right)(\nu<n)$ such that $S$ has undecidable word problem (see e.g. Davis [2]). We are going to define a finite extension $T^{*}$ of $T(K)$ and an effective map associating with every pair $\langle V, W\rangle$ of words in $\alpha_{1}, \alpha_{2}$ an $L$-sentence $\varphi$ such that $V=W$ holds in $S$ if and only if $T^{*}+\varphi$.

Let $A$ be a $p$-group and $a \in A$. Put $\tau(a)=\left\langle h(a), h(p a), h\left(p^{2} a\right)\right\rangle$ where $h$ is the $p$-height, 1.e. $h(x)=k$ if and only if $x \in p^{\kappa} A-p^{\kappa+1} A$. Put $\tau_{0}=\langle 0,2,7\rangle$, $\tau_{1}=\langle 0,3,6\rangle, \tau_{2}=\langle 0,4,5\rangle$. Note that for each pair $\langle i, j\rangle, i, j \leqslant 2, i \neq j, \tau_{i}$ has a component which is greater than the corresponding component of $\tau_{j}$.

For $j=1,2$ let $\varphi_{j}(x, y)$ be the $L$-formula

$$
\begin{aligned}
x=y=0 \vee \exists x^{\prime}, y^{\prime}\left(P\left(x^{\prime}\right) \& P\left(y^{\prime}\right) \& x=p^{3} x^{\prime} \& y\right. & =p^{3} y^{\prime} \\
\& \tau\left(x^{\prime}\right)=\tau_{0} \& \tau\left(y^{\prime}\right) & \left.=\tau_{j} \& h\left(x^{\prime}-y^{\prime}\right)=1\right) .
\end{aligned}
$$

Let $T^{*}$ be the theory obtained from $T(K)$ by adjoining axioms (i) and (ii) below.

Received by the editors March 26, 1975.

AMS (MOS) subject classifications (1970). Primary 02G05.

${ }^{1}$ Supported by Schweizerischer Nationalfonds. 


$$
\forall x\left(h(x) \geqslant 8 \rightarrow \exists ! y\left(h(y) \geqslant 8 \& \varphi_{j}(x, y)\right)\right), \quad j=1,2 .
$$

(i) simply says that $\varphi_{j}$ defines a function $f_{j}$ on the set of elements of height $\geqslant$ 8. Therefore every word $W\left(f_{1}, f_{2}\right)$ in $f_{1}, f_{2}$ defines a function $\overline{W\left(f_{1}, f_{2}\right)}$, and a definition of this function can easily be written down in terms of $\varphi_{1}, \varphi_{2}$.

$$
\forall x\left(h(x) \geqslant 8 \rightarrow \overline{V_{\nu}\left(f_{1}, f_{2}\right)}(x)=\overline{W_{\nu}\left(f_{1}, f_{2}\right)}(x)\right), \quad \nu<n .
$$

From (ii) it follows immediately that

$$
T^{*} \vdash \forall x\left(h(x) \geqslant 8 \rightarrow \overline{V\left(f_{1}, f_{2}\right)}(x)=\overline{W\left(f_{1}, f_{2}\right)}(x)\right)
$$

whenever $V\left(\alpha_{1}, \alpha_{2}\right)=W\left(\alpha_{1}, \alpha_{2}\right)$ holds in $S$. Since every countable semigroup can be embedded in the semigroup of endomorphisms of a countable vector space over the field $F$ with $p$ elements, the converse (and the theorem) clearly follow from

Claim. For any pair $g_{1}, g_{2}$ of endomorphisms of a countably infinite vectorspace $V$ over $F$ there exists a model $\langle A, B\rangle$ of $T(K)$ satisfying (i) such that $\left\langle V, g_{1}, g_{2}\right\rangle$ $\cong\left\langle p^{8} A, f_{1}, f_{2}\right\rangle$ where $f_{1}, f_{2}$ are defined by $\varphi_{1}, \varphi_{2}$.

Proof of Claim. Put $M=\{1,3,4,6,7,9\}$ and for $i \in M$ put $A_{i}$ $=\left(\mathbf{Z} / p^{i} \mathbf{Z}\right)^{(\omega)}, A=\oplus_{i \in M} A_{i}$, and let $\left(a_{i, k}\right)_{k \in \omega}$ be a basis of $A_{j}$. Identify $V$ with $p^{8} A=p^{8} A_{9}$ and let $a_{9, k} \in A_{9}$ such that $g_{j}\left(p^{8} a_{9, k}\right)=p^{8} a_{9, k}, j=1,2, k$ $\in \omega$. For $k \in \omega$ put

$$
\begin{aligned}
& b_{0, k}=p^{5} a_{9, k} \\
& +p a_{3, k}+a_{1, k} \\
& b_{1, k}=p^{5} a_{9, k}^{(1)}+p^{4} a_{7, k} \\
& +p^{2} a_{4, k} \\
& +a_{1, k} \\
& b_{2, k}=p^{5} a_{9, k}^{(2)} \\
& +p^{3} a_{6, k} \\
& +a_{1, k}
\end{aligned}
$$

and let $B$ be the subgroup of $A$ generated by all the $b_{j, k}$ 's. Note that $\tau\left(b_{j, k}\right)=\tau_{j}$. The important property of these generators is

$$
\text { if } b=\sum_{i \leqslant 2} \sum_{k \in \omega} r_{i, k} b_{i, k}, r_{i, k} \in \mathbf{Z} \text {, and } \tau(b)=\tau_{j} \text {, then }
$$$$
p \text { divides } r_{i, k} \text { for all }\langle i, k\rangle \text { such that } i \neq j \text {. }
$$

Assume, e.g., $\tau(b)=\tau_{1}$. Then $p$ divides $r_{0, k}$ because otherwise $h(p b)=2$, and $p$ divides $r_{2, k}$ because otherwise $h\left(p^{2} b\right) \stackrel{0}{=}$. The remaining two cases are similar.

Next we show that $\varphi_{j}\left(a, g_{j}(a)\right)$ holds in $\langle A, B\rangle$ for all $a \in p^{8} A, j=1,2$. This is clear if $a=0$. Assume $a=\sum_{k} r_{k} p^{8} a_{9, k} \neq 0,0 \leqslant r_{k}<p$. Put $x^{\prime}$ $=\sum_{k} r_{k} b_{0, k}, y^{\prime}=\sum_{k} r_{k} b_{j, k}$ and look at the definition of $\varphi_{j}$ and $B$.

It remains to show that $\langle A, B\rangle$ satisfies (i). Assume $\varphi_{1}\left(a, a_{1}\right)$ and $\varphi_{1}\left(a, a_{2}\right)$ both hold in $\langle A, B\rangle$ (the case $j=2$ is analogous). To show: $a_{1}=a_{2}$. Leaving the simpler case $a=0$ to the reader we assume $a \neq 0$. By the definition of $\varphi_{1}(x, y)$ there exist $b_{1}^{\prime}, b_{2}^{\prime}, b_{1}, b_{2} \in B$ such that for $j=1,2$

(1) $a=p^{3} b_{j}^{\prime}, a_{j}=p^{3} b_{j}$

(2) $\tau\left(b_{j}^{\prime}\right)=\tau_{0}, \tau\left(b_{j}\right)=\tau_{1}$,

(3) $h\left(b_{j}^{\prime}-b_{j}\right)=1$.

Write $b_{j}^{\prime}=\sum_{i \leqslant 2} \sum_{k \in \omega} r_{i, k}^{(j)} b_{i, k}, b_{j}=\sum_{i \leqslant 2} \sum_{k \in \omega} s_{i, k}^{(j)} b_{i, k}, r_{i, k}^{(j)}, s_{i, k}^{(j)} \in \mathbf{Z} .(*)$ and (2) imply 


$$
p \text { divides } r_{1, k}^{(j)}, r_{2, k}^{(j)}, s_{0, k}^{(j)}, s_{2, k}^{(j)} \text { for all } k \in \omega, j=1,2 .
$$

This together with (3) gives

$$
r_{0, k}^{(j)} \equiv s_{1, k}^{(j)}(\bmod p) \quad \text { for all } k \in \omega, j=1,2 .
$$

(1) and (4) imply

$$
a=\sum_{k} r_{0, k}^{(j)} p^{3} b_{0, k}, \quad a_{j}=\sum_{k} s_{1, k}^{(j)} p^{3} b_{1, k}, \quad j=1,2 .
$$

Combining the last two equations with $(5)$ we obtain $s_{1, k}^{(1)} \equiv s_{1, k}^{(2)}(\bmod p)$ for all $k$, and therefore $a_{1}=a_{2}$. This proves Theorem 1 .

REMARK. Although $T(K)$ is undecidable it is impossible to interprete number theory in it. This is a consequence of the fact that $T(K)$ is stable in the sense of Shelah [5] whereas number theory is unstable. Stability of $T(K)$ follows from [1] and the proof of Corollary 3 below.

2. Theories of modules. For any recursive ring $R$ with identity let $T_{R}$ denote the first order theory of $R$-modules in the language with nonlogical symbols 0 , ,$+ f_{r}(r \in R)$ (cf. Eklof-Sabbagh [3]). Theorem 1 can be used to prove undecidability of $T_{R}$ for various rings $R$.

COROLlaRy 3. There exist finite commutative rings $R$ such that $T_{R}$ is undecidable.

Proof. Put $R=R^{\prime}[X] /\left(X^{2}\right)$ where $R^{\prime}$ is the prime ring of characteristic $2^{9}$. If $M$ is an $R$-module then the pair $\mathfrak{A}_{M}=\langle\{m \in M \mid X m=0\}, X M\rangle$, considered as a pair of abelian groups, is a model of $T(K)$. Conversely assume $\langle A, B\rangle \vDash T(K)$. Let $B_{1}$ be an isomorphic copy of $B$ and define an endomorphism $X$ of $M=A \oplus B_{1}$ by $X a=0$ for $a \in A, X b_{1}=b$ for $b_{1} \in B_{1}$. Clearly this provides $M$ with an $R$-module structure, and $\mathfrak{A}_{M}=\langle A, B\rangle$. This gives a faithful interpretation of $T(K)$ in $T_{R}$, hence $T_{R}$ is undecidable.

Let $F$ be a finite field. The decidability proof for the theory of abelian groups given by Szmielew [6] applies also to $T_{F[X]}$. In contrast the next corollary shows that $T_{F[X, Y]}$ is undecidable.

COROLlary 4. If $R \neq 0$ is any recursive commutative ring then $T_{R[X, Y]}$ is undecidable.

Proof. Replacing $p$ by $X$ and making a few obvious changes in the proof of Theorem 1 we obtain that the theory of the class of structures $\langle M, N\rangle, M$ an $R[X]$-module and $N \subseteq M$ a submodule, is undecidable. By an argument similar to the one used in the proof of Corollary 3 it follows that $T_{R[X, Y]}$ is undecidable.

\section{REFERENCES}

1. W. Baur, $\aleph_{0}$-categorical modules, J. Symbolic Logic 40 (1975), 213-220.

2. M. Davis, Computability and unsolvability, McGraw-Hill Ser. in Information Processing and Computers, McGraw-Hill, New York, 1958. MR 23 \#1525.

3. P. C. Eklof and G. Sabbagh, Model-completions and modules, Ann. Math. Logic 2 (1970/71), no. 3, 251-295. MR 43 \#3105.

4. G. T. Kozlov and A. I. Kokorin, Elementary theory of abelian groups without torsion, with a 
predicate selecting a subgroup, Algebra i Logika 8 (1969), 320-334 = Algebra and Logic 8 (1969), 182-190. MR 41 \#3263.

5. S. Shelah, Stability, the f.c.p., and superstability: Model theoretic properties of formulas in first order theory, Ann. Math. Logic 3 (1971), no. 3, 271-362. MR 47 \#6475.

6. W. Szmielew, Elementary properties of Abelian groups, Fund. Math. 41 (1955), 203-271. MR $17,233$.

Seminar für Angewandte Mathematik der Universität Zürich, Freierstrasse 36, 8032 ZÜRICH, SWITZERLAND 